\title{
Diana Mistreanu
}

Université du Luxembourg/Université Paris-Est

\section{Exercices de médiocrité : le Manuel de peinture et de calligraphie (1977) de José Saramago} à travers le prisme de l'analyse transactionnelle

Il faut se connaître soi-même. Quand cela ne servirait pas à trouver le vrai, cela au moins sert à régler sa vie.

Pascal $^{1}$

Introduction : le texte et son contexte

Tosé de Sousa Saramago (1922-2010) n'a point

besoin d'introduction. Lauréat du Prix Camões (1995) et unique lauréat lusophone du Prix Nobel de Littérature (1998), il a réussi à faire résonner son nom dans des milieux où la littérature fictionnelle pénètre plus difficilement. Aussi s'est-il fait critiquer sur la scène politique, tant par le gouvernement du Portugal, pour son iconoclaste Évangile selon Jésus-Christ (1991)², que par les institutions de l'Union européenne ${ }^{3}$ et l'Église catholique,

\footnotetext{
${ }^{1}$ B. Pascal, Pensées et opuscules, Paris, Hachette, 1909, p. 345-346.

2 Le Premier Ministre Aníbal Cavaco Silva, avec l'aide du sous-secrétaire d'État Antonio de Sousa Lara, a ordonné de retirer ce titre de la liste proposée par le Portugal pour le Prix de littérature de l'Union européenne. ${ }^{3}$ Certains de ses ouvrages ont été interprétés comme des allégories de la décadence de l'Europe et de l'Union européenne. Ainsi, le roman Le Radeau de pierre (A jangada de pedra, 1986), publié à la veille de l'entrée du Portugal dans I'U. E., porte sur un cataclysme naturel qui frappe la péninsule ibérique et la sépare du continent ; il peut être lu comme un texte néo-conservateur sur l'américanisation de l'Europe occidentale. Saramago considère ce type de lecture comme réductrice. $C f$. "Saramago, José ", [dans :] V. E. Taylor et C.E. Winquist (dir.), Encyclopedia of Postmodernism, London-New-York, Routledge, 2004, p. 353-354, et D. J. Sadlier, The Portuguese Speaking Diaspora. Seven Centuries of Literature and the Arts, Austin, University of Texas Press, 2016, p. 209.
} 
prompte à protéger ses ouailles de l'athéisme fervent d'un personnage aussi illustre. Si ses prises de position politiques, corroborées par sa rhétorique extravagante et provocatrice, sont rapidement devenues notoires, cela n'a pas été le cas pour l'intégralité de son œuvre littéraire. Ainsi, le texte qui fait l'objet de notre analyse, à savoir le Manuel de peinture et de calligraphie (Manual de pintura e caligrafia), publié aux Éditions Moraes de Lisbonne en 1977, est longtemps resté en marge de l'attention critique, en raison notamment de sa traduction tardive, souvent ultérieure à d'autres romans de Saramago qui sont parus plus tard ${ }^{4}$. Malgré ce décalage, le texte reste, à la fois du point de vue stylistique et thématique, symptomatique pour l'ensemble de l'œuvre de l'auteur portugais. On y trouve déjà les marques de son écriture et son usage particulier de la ponctuation ${ }^{5}$, comme les longues phrases complexes séparées par des virgules et non par des points finaux, ainsi que les réflexions sur la condition humaine qui alimentent les thèmes majeurs de sa création, à savoir la recherche et la fragmentation de l'identité, les visions politiques de gauche ou l'existence humaine entre l'intime et le politique. De surcroît, le titre même est trompeur, car le texte n'est pas rédigé sous la

\footnotetext{
${ }^{4}$ Les lecteurs ont dû attendre douze ans pour que la première traduction voie le jour, en espagnol - géographie oblige (1989, par Basilio Losada). Elle a été suivie par la traduction allemande (1990, Maria Eduarda Alvelos), anglaise (1993, Giovanni Pontiero) et italienne (1996, Rita Desti), tandis que les traductions française (2000, Geneviève Leibrich), roumaine (2010, Georgiana Bărbulescu) et polonaise (2010, Wojciech Charchalis) ont été réalisées après que l'auteur a obtenu le Prix Nobel. Ajoutons que le roman n'a pas été publié aux États-Unis avant 2012 et qu'il n'est pas encore traduit en russe. En outre, l'histoire des traductions anglaises de Saramago est particulière, car elle a donné naissance à une touchante amitié entre l'écrivain et le traducteur. Pour plus de détails sur cette histoire et sur la réception de Saramago en Grande-Bretagne, consulter l'article de J.C. Sager, "Traducciones al inglés de las novelas de José Saramago creadas por Giovanni Pontiero y su recepción en Inglaterra », [dans :] Quaderns : Revista de Traducció, 2003, n 10, p. 111-120.

${ }^{5}$ Cf. V. Matos, "Les Portugais, I'lbérie et l'Europe ", [dans :] Pôle Sud. Revue de science politique de l'Europe méridionale, 2013, n³9, p. 99-110.
} 
forme d'un manuel, mais sous celle d'un journal et, comme José Belmonte Serrano et Pedro Guerrero Ruiz l'ont pertinemment remarqué, il s'inscrit dans la logique des futurs titres de Saramago, qui semblent annoncer plutôt des ouvrages non-fictionnels, des mémoriaux ou des essais ${ }^{6}$.

\section{Osons faire ce choix : littérature et psychothérapie}

Le texte consiste dans le journal intime du portraitiste H., qui passe par une crise existentielle et décide d'écrire afin d'apaiser ses frustrations professionnelles et personnelles. Cependant, au fur et à mesure que la rédaction avance, l'écriture prend des tournants inattendus et révèle à son auteur des informations précieuses sur sa propre personne, sur son rapport à son métier et sur ses relations avec autrui. Cet " apprentissage " de soi déclenche une nouvelle crise, positive, cette fois-ci, car elle permet au narrateur d'assumer sa vocation, qu'il vient de découvrir, lui donne la force de passer de la sexualité à l'amour, l'aide à sortir des schémas psychiques dont il était prisonnier et le détermine à vivre de manière libre et consciente - ou pour mieux dire, à s e vivre. Cela étant dit, notre article retracera les mécanismes psychiques qui sont à l'œuvre dans ce processus transformatif complexe, afin de mettre en lumière le chemin vers la connaissance et l'acceptation de soi, qui passe par l'écriture intime. Pour ce faire, nous proposons une approche psychothérapique de l'ouvrage, que nous interrogerons à travers

\footnotetext{
${ }^{6}$ J.B. Serrano et P.G. Ruiz, "La escripintura según José Saramago: Manual de pintura y caligrafía ", [dans :] Romance Quarterly, 2004, vol. 51, n० 4, p. 297. En outre, les deux auteurs ajoutent que le titre aurait contribué à ralentir la réception de l'ouvrage, avis également partagé par l'auteure d'une brève recension de ce texte parue dans la publication newyorkaise Library Journal : " Its misleading title, especially coming from an author known primarily as a journalist and translator, may have led to its neglect ". K.Gray, "Saramago, José. Manual of Painting and Calligraphy ", [dans :] Library Journal, 2012, vol. 137, n 4, p. 89-90.
} 
le prisme de l'analyse transactionnelle7. Certes, les contraintes rédactionnelles ne nous permettent que d'effleurer le sujet, et de ce fait, nous avons choisi de nous limiter à ce que les outils de l'analyse transactionnelle révèlent sur le narrateur du texte. Ce que nous souhaitons, en procédant ainsi, est d'ouvrir un chemin qui nous semble fécond et prometteur : celui de l'exploration des textes littéraires en articulant linguistique et analyse transactionnelle. Qui plus est, malgré l'absence - à notre connaissance - d'études qui proposent ce type d'approche, il nous semble qu'elles seraient non seulement légitimées, mais aussi envisagées et encouragées par le fondateur même de l'analyse transactionnelle, selon lequel il faudrait " des années d'études pour maîtriser les subtilités de la communication verbale $»^{8}$. Or, quel miroir des interactions entre les jeux psychologiques et les subtilités communicationnelles est-il plus vaste et plus clair que la littérature?

\section{L'analyse transactionnelle - éclairons quelques concepts}

L'analyse transactionnelle (A.T.) est apparue aux États-Unis à la fin des années 1950. Son nom désigne une théorie psychodynamique fondée sur la réinterprétation que son fondateur, le psychiatre d'origine canadienne Eric Berne (1910-1970), a faite de la psychanalyse ${ }^{9}$. Largement

7 L'objectif de notre article étant d'apprendre ce que cette théorie psychodynamique peut révéler sur un texte littéraire, nous reléguons expressément au second plan l'analyse stylistique du texte. Le texte se prête, bien évidemment, à de nombreux autres types de lecture appuyés sur d'autres méthodologies.

${ }^{8}$ A. le Guernic, "AT et linguistique dans le modèle des transactions ", [dans :] Actualités en analyse transactionnelle, 2008, vol. 2, n 126, p. 56.

${ }^{9}$ Dans un de ses premiers livres, Berne utilise le parcours de Freud pour le diagnostiquer et pour illustrer ainsi un des types humains. Cf. E. Berne, "Sigmund, or If you can't do it one way, try another ", [dans :] Idem, What Do You Say After You Say Hello, New York, Groove Press Inc., 1972, p. 225. Pour une présentation succincte de la vie de Berne, de sa rupture 
utilisée aujourd'hui en psychothérapie partout dans le monde, I'A.T. a un but pratique : à l'instar de la psychanalyse, elle vise à accéder à l'inconscient du sujet pour identifier et, par la suite, régler les dysfonctions, les dysharmonies, les traumas et tout autre facteur qui agit dans l'ombre et diminue la qualité de la vie. À la différence de la psychanalyse, cependant, son point de départ n'est pas l'examen du soi fait par le patient, mais des relations sociales de celui-ci, qui sont considérées comme l'outil d'accès à son inconscient et pour la théorisation desquelles Berne propose un schéma conceptuel dont la terminologie est empruntée aux contes de fées et aux mythes de la Grèce antique. II s'agit donc d'une théorie complexe qui a évolué depuis sa création $^{10}$, de sorte qu'il nous est impossible de l'exposer ici de manière exhaustive. Aussi avons-nous choisi d'appuyer notre analyse sur trois concepts uniquement, qui se trouvent au cœur de I'A.T. et qui nous semblent les plus pertinents pour notre démarche.

Le premier concept est celui des " états du Moi ", considérés comme les principaux composants de la personnalité, pour lesquels Berne propose le modèle tripartite Parent-Adulte-Enfant. Chaque état représente un système cohérent de pensées, d'émotions et de comportements associés ${ }^{11}$, dissociables de l'âge biologique, activés alternativement, en fonction de la situation. Le Parent réside dans les comportements internalisés d'un des parents ou d'une des figures parentales dominantes et il correspond au domaine de l'appris, c'est-à-dire aux règles, aux devoirs et aux interdits. Cette instance normative est activée, par exemple, quand le sujet devient lui-même parent. L'Adulte, quant à lui, est caractérisé par

avec la psychanalyse et la création de l'A.T., consulter l'article de E. Alleysson, "Biographie d'Eric Berne, fondateur de l'analyse transactionnelle ", [dans :] Actualités en analyse transactionnelle, 2010, vol. 3, n० 135, p. 1-5.

${ }^{11}$ E. Berne, What Do You Say After You Say Hello, op. cit., p. 11. 
l'analyse et l'évaluation objective des faits, par la neutralité, et il correspond au domaine du raisonnement. L'Enfant, enfin, relève du monde des émotions, de l'affectivité et des sentiments. Selon Berne, chaque personne porte en elle un enfant qui sent et réfléchit de la même manière qu'elle le faisait dans une étape de son enfance, située d'habitude entre deux et cinq ans. Un des objectifs de I'A.T. en tant que pratique psychothérapeutique est que l'individu comprenne et accepte cet enfant, puisqu'il l'accompagnera pendant toute sa vie, mais aussi parce qu'il représente ce qu'il a de plus précieux. En outre, ce sont les interactions, appelées aussi " transactions ", entre les états du Moi de différentes personnes qui sont à l'origine du nom de cette théorie.

Le deuxième concept de I'A.T. est le triangle dramatique de Stephen Karpman, qui est un modèle d'étude des interactions sociales. En effet, Karpman a postulé trois types de rôles, à savoir celui de victime, de sauveteur et de persécuteur, qui gouvernent nos rapports aux autres et qui deviennent manifestes notamment dans des situations conflictuelles ${ }^{12}$. Le persécuteur, appelé aussi bourreau, est par excellence agressif. II dévalorise et ironise, il exerce un abus verbal, émotionnel ou physique sur la victime, qui, elle, subit, se plaint et attire ainsi le sauveteur. À son tour, celui-ci est à la recherche de bourreaux pour justifier son existence et de victimes pour gratifier son narcissisme. Une situation de crise est, dans cette optique, une situation de redistribution des rôles : une victime qui se rebelle devient souvent un persécuteur, tandis que le sauveteur devient facilement victime. Selon Karpman, nous passons environ trois quarts de notre temps dans un de ces rôles, qui nous a été assigné pendant les premières années de l'enfance.

12 S. Karpman, " Fairy tales and script drama analysis ", https://www.karpmandramatriangle.com/pdf/DramaTriangle.pdf. 
C'est ici qu'intervient la troisième notion de l'A.T., celle de scénario de vie, ou de script. Selon Berne, ce concept désigne un plan que nous internalisons pendant "les années plastiques ${ }^{13}$, à savoir les six premières années de l'enfance, et que nous chercherons inconsciemment à mener à bien plus tard dans la vie. Étant le résultat de la programmation parentale, par le biais de laquelle l'enfant acquiert sa vision de soi et du monde, le scénario commence à se cristalliser très tôt, dès la période de l'allaitement ${ }^{14}$. Berne cite à cet égard l'exemple déstabilisateur de ce qu'il appelle "the gallows laugh", à savoir la pratique des criminels londoniens du XVIII siècle qui, juste avant leur pendaison, devenaient de véritables maîtres de l'humour public et amusaient la foule venue les voir par des blagues et des épigrammes. II ne s'agissait pas d'un simple défi lancé à la mort, car quelque chose de plus profond était à l'œuvre : ils étaient en train d'accomplir l'injonction parentale suprême - Tu finiras pendu comme ton père, mon petit !- et de mener ainsi leur scénario à son terme. Ils mouraient en paix avec leurs mères, donc contents ${ }^{15}$.

Le fondateur de I'A.T. a identifié trois typologies de scénarios, à savoir celui du vainqueur, appelé aussi Prince, celui du vaincu, ou de la Grenouille, et, enfin, celui du nonvainqueur. Le vainqueur est quelqu'un qui réussira à accomplir ce qu'il s'est proposé de réaliser ; il aura du succès parce qu'il sait ce qu'il fera s'il perd, sans en parler à personne, et parce qu'il ne cesse jamais d'essayer. II dit des phrases telles que " J'ai fait une erreur, mais je ne la répéterai pas " et "Maintenant, je connais le bon chemin ". À l'autre bout du spectre se trouve le vaincu, qui est en proie à l'échec. À la différence du vainqueur, il se caractérise par le fait qu'il ne sait pas ce qu'il fera s'il perd,

${ }^{13}$ E. Berne, What Do You Say After You Say Hello, op. cit., p. 97.

14 Ibidem, p. 83.

15 Ibidem, p. 196. 
mais il parle de ce qu'il fera après son succès. II se distingue également par l'emploi fréquent des expressions conditionnelles ("Oh, si seulement... ", " J'aurais pu/ dû... ", "Oui, mais... ", etc.). Quant au non-vainqueur, il se situe entre les deux : tantôt il gagne, tantôt il perd, mais il ne s'agit jamais de choses importantes, puisqu'il ne prend pas de risque. Il est souvent une personne qui travaille beaucoup, non pas pour gagner, mais parce que cela lui permet de continuer son existence : c'est le hard-worker, le fonctionnaire au service des grands, qui se contente de sa position, de son travail et de ce que la vie lui offre sans essayer de le changer. Ses phrases-types sont "Au moins, je n'ai pas... " et " Je suis reconnaissant pour ce que je possède déjà ".

Si les états du Moi se situent sur le plan intrapersonnel et le triangle de Karpman développe un schéma interpersonnel, tous les deux doivent être compris à travers le prisme du scénario de vie. Il convient de souligner que ce dernier est extrêmement puissant justement parce qu'il est inconscient et qu'il contient en lui la programmation des plus importants aspects de la vie du sujet, comme la manière dont il utilisera son temps, le métier qu'il choisira d'exercer, sa vision de l'amour et de la sexualité, le genre de partenaire qu'il cherchera une fois devenu adulte, voire les maladies qui l'atteindront et la façon dont il mourra. Ainsi, seul un désastre peut transformer un Prince éternel en Grenouille et seul un miracle peut transformer une Grenouille condamnée en un Prince ${ }^{16}$. Le caractère si détaillé de cette programmation pose, bien évidemment, le problème de la liberté individuelle, qui paraît annulée par des mécanismes de prédestination parentale. En réalité, selon Berne, l'enfant naît libre et ces mécanismes qui se cristallisent et restreignent sa liberté dès l'enfance peuvent être démantelés. La fidélité aux ordres de la mère, comme

16 Ibidem, p. 84. 
dans le cas des criminels londoniens, n'est pas une fatalité, car elle n'est pas donnée, mais choisie. Autrement dit, si le scénario est conçu de manière à durer pour la vie, le fait de le suivre, en revanche, ne l'est pas, et c'est dans cette brèche fragile entre choix et prédétermination qu'intervient le thérapeute, dont le rôle est d'aider le patient à identifier son scénario, à en devenir conscient et, par conséquent, à ne plus se sentir condamné à le mener à bien, voire à devenir capable de le modifier. Cela équivaut à la guérison du patient, ou, traduit dans la terminologie de Jung, à son individuation, à la suite de laquelle ont lieu des changements qualitatifs observables. $\mathrm{Au}$ vu de ces considérations, examinons à présent comment ce triple modèle conceptuel constitué des états du Moi, du triangle dramatique de Karpman et de la notion de scénario de vie fonctionne en tant qu'outil d'exploration littéraire.

\section{L'écriture intime au miroir de l'A.T.}

Selon Bernard Lahire, l'écriture intime ${ }^{17}$ est une forme de travail symbolique sur soi, acte qui « n'est pas anodin

\footnotetext{
17 Le texte de Saramago est une fiction à la première personne, sans identité entre l'auteur et le narrateur, ce dernier étant une instance fictive. De ce fait, l'ouvrage n'est pas une autobiographie - selon la définition que Philippe Lejeune confère à ce concept, qui suppose l'identité entre, d'une part, l'auteur, qui est une personne réelle, et de l'autre, le narrateur et le personnage central du récit. II n'est pas non plus une autofiction, dans l'acception de Serge Doubrovsky, pour la même raison : le principe des trois identités n'y est pas respecté. Saramago a débuté à 25 ans, et le travail de traducteur et de journaliste qu'il a fait avant de pouvoir vivre de sa plume indique une prise de connaissance précoce de sa vocation pour le monde des lettres. Certes, il est fort possible qu'il se soit inspiré de son propre vécu pour écrire cet ouvrage, et certains éléments biographiques légitiment l'exploration de cette piste de recherche. Néanmoins, l'absence des marqueurs textuels de l'autobiographie et de l'autofiction, ainsi que celle d'éventuelles déclarations de la part de l'auteur à ce sujet nous ont déterminée à ne pas nous occuper, pour l'instant, de cette grille de lecture. Cf. P. Lejeune, Le Pacte autobiographique, Paris, Seuil, 1975 et V. Colonna, L'autofiction. Essai sur la fictionalisation de soi en littérature, thèse de doctorat sous la direction de Gérard Genette, Paris, EHESS, 1989.
} 
ou banal $»^{18}$. Or, ses causes et ses conséquences ne le sont pas non plus. La recherche en sociologie montre, en effet, que le passage à l'écriture se réalise souvent dans des moments de rupture biographique ou identitaire tels le divorce, le décès d'une personne proche, l'expérience du déracinement, le service militaire, etc., ou dans des moments qui balisent le cycle de vie, comme l'adolescence, la naissance du premier enfant, constitutive du statut du parent, ou le départ en retraite ${ }^{19}$. Le journal peut ainsi être " un catalogue de situations vécues écrites, relues et retravaillées " qui devient le lieu de la "réflexivité sur soi, son passé et son avenir ${ }^{20}$. Rappelons, par exemple, que le narrateur du roman La familia de Pascual Duarte (1942) du Prix Nobel espagnol Camilio José Cela commence à rédiger ses mémoires en prison, après avoir été condamné à mort.

Dans le cas du personnage-narrateur du Manuel..., qui s'auto-désigne par l'initiale $\mathrm{H}$., ce qui provoque le passage à l'écriture est la rencontre avec S., un patron d'entreprise qui désire se faire peindre. Cette rencontre qui, a priori, ne devrait présenter rien de particulier, puisque le narrateur affirme avoir une multitude de clients qui font partie de la même catégorie socio-professionnelle, bouleverse $\mathrm{H}$. et déclenche un long processus d'introspection, dont le journal qu'il vient d'entamer devient le miroir. Qui plus est, le portraitiste ne change pas seulement de medium d'expression, mais il essaie, pour la première fois, $d^{\prime}$ 'innover ses pratiques picturales. Il entame ainsi deux portraits de S., l'un académique, l'autre, une œuvre plus personnelle, dans laquelle il s'éloigne des normes, mais qui s'avère un échec du point de vue esthétique. Aussi le texte qu'il est en train d'écrire devient-il une accumulation

\footnotetext{
${ }^{18}$ B. Lahire, « De la réflexivité dans la vie quotidienne : journal personnel, autobiographie et autres écritures de soi ", [dans :] Sociologie et sociétés, 2008, vol. 40, n², p. 166.

${ }^{19}$ Ibidem, p. 173.

20 Ibidem.
} 
de marqueurs d'émotions négatives telles la honte et la colère, ainsi que le lieu d'expression de ses angoisses :

J'ai presque cinquante ans, [l'âge] de la vieillesse approche, et soudain, je le répète, il m'est devenu intolérable d'échouer, de ne pas savoir, de continuer à gesticuler dans l'obscurité, d'être un automate qui rêve toutes les nuits de se débarrasser de la bande perforée de son programme : un long ténia qui fut la seule vie qui a existé entre les circuits et les transistors. Si vous me demandiez si j'aurais pris une décision semblable même si S. n'était pas apparu dans ma vie, je serais incapable de vous répondre. ${ }^{21}$

Le passage de $\mathrm{H}$. à de nouveaux modes d'expression est le corollaire d'une décision existentielle prise à la suite de la rencontre qu'il a faite, à savoir celle de démonter les mécanismes qui l'empêchaient de changer sa vie. Les figures de style qu'il utilise sont révélatrices à cet égard. L'isotopie des ténèbres et de l'inconnu (" obscurité ", "rêve ", " nuits ") renvoie à la partie de lui-même qu'il ne connaît pas, mais dont la connaissance est indispensable pour vivre consciemment et pleinement, tandis que " l'automate ", à qui font écho les " circuits et les transistors ", est une référence claire à l'idée de programmation, qui dans le paradigme de l'A.T. équivaut au scénario de vie. Or, comme le précise Anne Clotilde Abécassis Ziégler, le scénario, qui est si intimement et profondément inscrit en nous - y compris au niveau biologique, dans notre système nerveux - peut néanmoins être repéré, car il " réside dans les métaphores que la personne utilise ${ }^{22}$. Ainsi, la référence au ténia en dit long. Le ténia est un ver parasite, solitaire et d'une dimension remarquable - l'adulte mesure entre 4 et 10 mètres de longueur. Dans ce contexte, l'emploi du déterminant " long » (" un long ténia »), qui pourrait

$21 \mathrm{~J}$. Saramago, Manuel de peinture et de calligraphie, G. Leibrich (trad.), Paris, Seuil, 2000, p. 20. Pour les citations suivantes provenant de cet ouvrage, nous emploierons la référence $M P C$.

22 A.C.A. Ziégler, "Le point sur... la scène de scénario ", [dans :] Actualités en analyse transactionnelle, 2010, vol. 4, n 136, p. 65. 
facilement être qualifié de pléonastique s'il était utilisé dans une autre circonstance, hyperbolise les traits de l'animal. De plus, cette métaphore revient sous d'autres formes dans le journal, par exemple lorsqu'il parle de son travail, qu'il exécute " anesthésié et indifférent " (MPC, 15) ou de la réception critique de ses toiles, qui le fait considérer qu'il s'est éternisé dans un " état larvaire " (MPC, 10). Le texte ne contient pas d'éléments précis sur le scénario de vie du narrateur - et rien n'est plus naturel, puisque la narration se fait à la première personne et que la plupart des personnes, à moins d'avoir suivi une formation psychothérapeutique, ignorent l'existence de leur scénario -, de manière que cette identification animale en est une marque importante. Elle met en lumière deux caractéristiques puissantes de l'image inconsciente - que le narrateur a de lui-même, à savoir le sentiment d'être un parasite et la condamnation à la solitude. Ce sont ces deux éléments qui le " paralysent " dans l'automate. S'il parvient à en prendre conscience, la déprogrammation devient possible. Si ce n'est pas le cas, ces éléments l'accompagneront jusqu'à sa tombe.

Malgré le poids symbolique de la métaphore animale, ne nous précipitons pas de conclure que $H$. possèderait un scénario de type " grenouille ", c'est-à-dire un scénario de vaincu. Ne nous laissons pas non plus tromper par la multitude de passages où il déplore sa médiocrité artistique ou décrit l'inertie de sa relation, essentiellement sexuelle, avec Adelina, qui le fréquente de temps en temps - nous y reviendrons. En effet, il existe dans le texte plusieurs éléments qui relèvent du scénario du nonvainqueur, qui, selon Berne, n'est ni prince ni grenouille, travaille assidûment et se contente du peu qu'il a. Le premier réside dans la métaphore de l'automate que nous avons citée ci-dessus, car si elle renvoie à l'idée de scénario, comme nous venons de l'affirmer, elle réfère aussi à une activité monotone, répétitive et perpétuelle, qui correspond à la manière dont $\mathrm{H}$. décrit son travail : 
Je fais tout cela sans plaisir, parce que cela figure parmi les préceptes, protégé par l'indifférence dont la critique m'a entouré à la façon d'un cordon sanitaire, protégé aussi par l'oubli où je suis tombé peu à peu parce que je sais que le tableau ne sera pas montré dans des expositions ou des galeries. [...] J'ai plus de travail qu'il ne me faut. [...] Mais ce que je fais n'est pas de la peinture. (MPC, 11)

Remarquons ici l'emploi de la métaphore du cordon sanitaire, qui fait écho à celle du ténia et qui renforce les traits dévalorisants de l'image de soi - car on n'entoure d'un cordon sanitaire que ce qui est malpropre et nuisible. Le second élément qui montre que $\mathrm{H}$. est dominé par un scénario de non-vainqueur découle de son attitude par rapport au journal qu'il est en train de rédiger. Selon Berne, la vainqueur dispose toujours d'un plan de secours, car c'est ainsi qu'il réussit dans la vie, mais il n'en parle jamais, tandis que le vaincu discute souvent de ce qu'il fera après son succès, qui n'advient pourtant pas. H., en ce qui le concerne, n'a aucun plan pour l'avenir au moment où il commence son journal, et il ne parle pas non plus de ce qu'il fera si l'écriture s'avère un autre insuccès :

je me refuse à penser à ce que je ferai si cet écrit était un échec lui aussi, si désormais toiles vides et feuilles blanches étaient pour moi un univers situé sur une orbite à des millions d'années lumières où je ne pourrai pas tracer le moindre signe. (MPC, 9)

Cette non-projection dans l'avenir indique que $\mathrm{H}$. est un non-vainqueur.

La rencontre avec S. révèle aussi un autre trait de la psychologie du narrateur, à savoir sa position dans le triangle dramatique de Karpman. À un détail près, il serait légitime pour le lecteur de considérer que ce personnage est dans le rôle de victime, dans un schéma où "les critiques ", instance impersonnelle évoquée à plusieurs reprises, correspondraient au persécuteur. Le haut degré de généralisation, comme dans ce cas où "les critiques" semblent désigner tous les critiques d'art existants, indique d'habitude une projection, à savoir un mécanisme de défense qui expulse de soi et perçoit dans un autre tout 
ce que l'esprit " ressent comme douloureux ou déplaisant ${ }^{23}$. Aussi est-il fort possible que $\mathrm{H}$. projette effectivement son " juge interne " - qui lui révèle constamment son infertilité créatrice - sur les critiques, en hyperbolisant et réitérant des remarques et reproches ponctuels qu'un ou deux critiques aurait faits dans le passé. Nous pourrons spéculer ici que $H$. est aux prises avec son Parent, l'instance à qui incombent les jugements de valeur, et qu'il essaie de l'éloigner de lui au lieu de l'accepter et de l'intégrer. Certes, cela ne reste qu'une hypothèse et n'annule en rien le fait que $H$. se perçoit aussi comme une victime, même si le bourreau réel, qui réside dans les attentes qu'il a de lui-même, corroborées par la non-acceptation de soi, est en sécurité, escamoté et protégé par une quantité de tortionnaires imaginaires. Cependant, son interaction avec $S$. révèle le fait qu'il passe le plus clair de son temps dans le rôle du sauveteur. Il est temps de préciser ici la manière dont $\mathrm{H}$. perçoit ses clients. Pour lui, ce sont des hommes riches, mais dépourvus de culture humaniste, des snobs dont le désir ardent de s'immortaliser par le biais du portrait éveille son mépris. Devant ces ignorants esclaves de l'argent, son manque de talent et son statut social et professionnel sont réhabilités symboliquement par ses connaissances littéraires et artistiques - ce qui, il va sans dire, est la preuve d'un orgueil aveugle. De plus, il a une attitude messianique par rapport à son métier :

Je me dis parfois et je me persuade que je suis le seul portraitiste qui reste et qu'après moi on ne perdra plus son temps en poses fatigantes [...]. Je me plais à penser que je cultive un art mort grâce auquel, par le biais de ma faillibilité, les gens croient fixer une certaine image agréable d'eux-mêmes, [...] image d'une éternité qui ne commence pas seulement quand le portrait est terminé, mais qui vient d'avant, de toujours, comme une chose qui a toujours existé simplement parce qu'elle existe en cet instant, une éternité qui va vers le néant. (MPC, 12)

${ }^{23} \mathrm{H}$. Chabrol, "Les mécanismes de défense ", [dans :] Recherche en soins infirmiers, 2005, vol. 3, n 82 , p. 31-42. 
" L'éternité » dont il question dans ce passage n'est possible que grâce à lui. Dans cette optique, avoir des clients gratifie son égo, car - au moins dans sa vision du monde - il accomplit un des actes les plus importants de leur vie. Or, l'attitude de S. fait voler ce fantasme en éclats. Non seulement il ne s'avère pas reconnaissant envers le peintre - et le sauveteur se nourrit de la reconnaissance des victimes -, mais il lui avoue que la décision de se faire peindre n'appartient pas à lui, mais à sa mère et au conseil d'administration de son entreprise, ce qui intensifie la crise du narrateur, qu'il exprime en ces termes :

Quand S. m'a dit en riant que son portrait était peint sur décision du conseil d'administration, volonté de sa mère et acquiescement de sa part, je suis demeuré immobile devant le chevalet, bras tendu, en suspens, regardant le pigment se déplacer lentement au bout de mon pinceau, viscère liquide soudain coupé de sa racine, mais encore palpitant, comme une queue de lézard ou la moitié restante d'un ver de terre aveugle. J'ai détesté $S$. pour m'avoir donné le sentiment d'être si malheureux, si irrémédiablement inutile, si peintre sans peinture [...]. Nous rêvons tous un jour de sauver quelqu'un de la noyade, et moi, après avoir nagé du mieux que je pouvais, j'avais entre les bras un bonhomme en plastique avec un masque railleur et un mécanisme interne siffleur. (MPC, 50)

Ce passage est significatif, car il contient les traits majeurs de la psychologie du personnage. Notons la récurrence de l'image de soi dévalorisante exprimée par l'isotopie animale. Les termes employés font écho à la métaphore du ténia, mais qui, cette fois-ci, revient avec plus de violence, car la "queue de lézard " de même que la " moitié d'un ver de terre aveugle " renvoient à l'idée de mutilation et rendent compte de la rupture psychique opérée par les mots de S. L'hypostase de sauveteur apparaît aussi, et de façon très claire, accompagnée par une autre généralisation - " nous rêvons tous un jour de sauver quelqu'un ". L'emploi du "nous " et du quantificateur " tous " au lieu de " je »/«moi " relève d'une projection exprimée linguistiquement par la 
généralisation, qui est, comme nous l'avons montré, l'un des mécanismes de défense à l'œuvre chez $\mathrm{H}$.

Dans la plupart des cas, $\mathrm{H}$. parle de lui à la première personne, selon les règles du journal intime, de manière que l'emploi du pronom personnel " je » est fréquent. Selon Benveniste, "quand l'émetteur du message dit "Je", il se désigne en disant "Je" et il dit quelque chose sur le compte de "Je" ${ }^{24}$. Dans le paradigme de l'A.T., ce pronom désigne, sur le plan psychique, les états du Moi entité tripartite - ce qui pose le problème du référent de "Je ", qui est tantôt l'Adulte, tantôt l'Enfant ou le Parent. Les éléments que nous avons repérés dans le passage cité ci-dessus, ainsi que dans d'autres fragments auxquels nous avons fait référence, indiquent que l'état du Moi qui est le plus actif chez le narrateur est l'Enfant. Certes, ses longues réflexions semblent, en apparence, appartenir à un adulte. Cependant, elles sont parsemées d'émotions et de sentiments intenses qu'il ne sait pas gérer et qui le prennent au dépourvu, et le registre de l'affectivité appartient surtout à l'Enfant. II en va de même pour ses mécanismes de défense, puisqu'un Adulte fonctionnel est capable d'évaluer chaque situation séparément, sans recourir à des projections ni à des généralisations. De plus, $H$. éprouve un besoin avide de reconnaissance, et $s^{\prime}$ il a appris à se passer de celle des "critiques ", le manque de reconnaissance de la part de ses clients s'avère douloureux. La source de ce besoin, ainsi que celle de la douleur, est, bien sûr, l'Enfant, un Enfant incompris et méconnu.

Conclusions : un message d'espoir?

Nous avons fait le point, dans cet article, sur ce que le texte de l'ouvrage révèle concernant la psychologie du

${ }^{24}$ É. Benveniste paraphrasé par A. le Guernic, « AT et linguistique dans le modèle des transactions ", op. cit., p. 55. 
personnage-narrateur au moment où celui-ci entame son journal. Pour ce faire, nous nous sommes appuyées sur les outils d'exploration fournis par l'analyse transactionnelle telle qu'elle a été conçue par Eric Berne. Nous avons ainsi montré que le scénario de vie, si insidieux soit-il, est néanmoins repérable par le biais des expressions linguistiques récurrentes, et que le narrateur $\mathrm{H}$. est dominé par un scénario de non-vainqueur, qui le détermine à travailler avec acharnement, à se contenter de ce qu'il possède et à ne pas procéder à des changements significatifs dans sa vie. Nous avons repéré aussi les marqueurs d'une image de soi dévalorisée, qui fait partie du scénario, ainsi que du rôle qu'il occupe dans le triangle dramatique de Karpman, qui est celui de victime par rapport aux "critiques ", et de sauveteur par rapport à ses clients. L'un des mécanismes de défense qui maintient actifs le scénario et la position dans le triangle dramatique a également été évoqué, à savoir la projection, exprimée linguistiquement par des généralisations. Nous avons, enfin, identifié l'état du Moi qui est le référent de la plupart des occurrences du pronom " je ", c'est-à-dire I'Enfant. Ces éléments sont la source de multiples dysfonctionnements qui empêchent le personnage de se réjouir de sa vie. Cependant, ils ne sont pas immuables. En effet, le personnage se transforme au fur et à mesure qu'il rédige son journal, car le processus d'écriture, qui l'aide à prendre conscience de soi, est thérapeutique. Dans ce sens, l'ouvrage s'avère, malgré tout, un texte à la fois remarquablement réaliste - car l'auteur fait preuve d'une connaissance minutieuse du psychisme humain - et lumineux, parce qu'il montre que même à des âges ou dans des états où rien ne semble davantage définitif, des changements profonds sont possibles. À condition, certes, de se connaître soi-même. 


\section{bibliographie}

Alleysson E., " Biographie d'Eric Berne, fondateur de l'analyse transactionnelle ", [dans :] Actualités en analyse transactionnelle, 2010, vol. 3, n० 135 .

Berne E., What Do You Say After You Say Hello, New York, Groove Press Inc., 1972.

Chabrol H., "Les mécanismes de défense ", [dans :] Recherche en soins infirmiers, 2005, vol. 3, n 82.

Colonna V., L'autofiction. Essai sur la fictionnalisation de soi en littérature, thèse de doctorat sous la direction de Gérard Genette, Paris, EHESS, 1989.

Gray K., "Saramago, José. Manual of Painting and Calligraphy ", [dans :] Library Journal, 2012, vol. 137, n 4.

Karpman S., " Fairy tales and script drama analysis ", https://www.karpmandramatriangle.com/pdf/DramaTriangle.pdf.

Lahire B., " De la réflexivité dans la vie quotidienne : journal personnel, autobiographie et autres écritures de soi ", [dans :] Sociologie et sociétés, 2008, vol. 40, n².

Le Guernic A., " AT et linguistique dans le modèle des transactions ", [dans :] Actualités en analyse transactionnelle, 2008, vol. 2, nº 126.

Lejeune P., Le Pacte autobiographique, Paris, Seuil, 1975.

Matos V., "Les Portugais, I'Ibérie et l'Europe ", [dans :] Pôle Sud. Revue de science politique de l'Europe méridionale, 2013, vol. 2, n 39.

Pascal B., Pensées et opuscules, Paris, Hachette, 1909.

Rath I., "Les phases de développement de l'analyse transactionnelle, d'Eric Berne à nos jours ", [dans :] Actualités en analyse transactionnelle, 2010, vol. 3, no 135.

Sadlier D. J., The Portuguese Speaking Diaspora. Seven Centuries of Literature and the Arts, Austin, University of Texas Press, 2016.

Sager J.C., "Traducciones al inglés de las novelasde José Saramago creadas por Giovanni Pontiero y su recepción en Inglaterra », [dans :] Quaderns : Revista de Traducció, 2003, nº 10.

Saramago J., Manuel de peinture et de calligraphie, G. Leibrich (trad.), Paris, Seuil, 2000.

Serrano J.B., Ruiz P.G., " La escripintura según José Saramago: Manual de pintura y caligrafía ", [dans :] Romance Quarterly, 2004, vol. 4, n 51.

Taylor V.E., Winquist C.E. (dir.), Encyclopedia of Postmodernism, London-New-York, Routledge, 2004.

Ziégler A.C.A., "Le point sur... la scène de scénario ", [dans :] Actualités en analyse transactionnelle, 2010, vol. 4, nº 136.

\section{abstract}

Exercises of Mediocrity: Saramago's Manual of Painting and Calligraphy through the Prism of Transactional Analysis

Albeit symptomatic of both Saramago's writing style and philosophical outlook on life, his Manuel de peinture et de calligraphie (1977), which is 
the private diary of a fictional painter whose gradual process of getting to know himself changes his life for the better, has not gained critical attention until recent years. Our paper discusses the psychology of the narrator and aims to unveil the mechanisms at work in his initial state of evolution, mechanisms that make him lead a frustrating intimate and professional life. The text is explored through the prism of Eric Berne's transactional analysis theory, and it pinpoints the character's states of the Ego, his place in Stephan Karpman's drama triangle, as well as various elements of his life script.

\section{keywords}

writing the Self, states of the Ego, script, drama triangle

\section{diana mistreanu}

Diana Mistreanu est chercheur en formation doctorale à l'Université du Luxembourg, en cotutelle avec l'Université Paris-Est, sous la direction de Madame le Professeur Sylvie Freyermuth et de Madame le Professeur Thanh-Vân Ton-That. Sa thèse porte sur l'œuvre d'Andreï Makine. Elle est également traductrice littéraire et membre fondateur du Cercle nomade des traductions littéraires de la Faculté des langues et littératures étrangères de l'Université de Bucarest. 